\title{
Neimark-Sacker Bifurcation of a Two-Dimensional Discrete-Time Chemical Model
}

\author{
A. Q. Khan \\ Department of Mathematics, University of Azad Jammu and Kashmir, Muzaffarabad 13100, Pakistan \\ Correspondence should be addressed to A. Q. Khan; abdulqadeerkhan1@gmail.com
}

Received 27 February 2020; Revised 18 June 2020; Accepted 22 June 2020; Published 6 August 2020

Academic Editor: Kishin Sadarangani

Copyright (c) 2020 A. Q. Khan. This is an open access article distributed under the Creative Commons Attribution License, which permits unrestricted use, distribution, and reproduction in any medium, provided the original work is properly cited.

In this paper, the local dynamics and Neimark-Sacker bifurcation of a two-dimensional glycolytic oscillator model in the interior of $\mathbb{R}_{+}^{2}$ are explored. It is investigated that for all $\alpha$ and $\beta$, the model has a unique equilibrium point: $P_{x y}^{+}\left(\left(\alpha / \beta+\alpha^{2}\right), \alpha\right)$. Further about $P_{x y}^{+}\left(\left(\alpha / \beta+\alpha^{2}\right), \alpha\right)$, local dynamics and the existence of bifurcation are explored. It is investigated about $P_{x y}^{+}\left(\left(\alpha / \beta+\alpha^{2}\right), \alpha\right)$ that the glycolytic oscillator model undergoes no bifurcation except the Neimark-Sacker bifurcation. Some simulations are given to verify the obtained results. Finally, bifurcation diagrams and the corresponding maximum Lyapunov exponent are presented for the glycolytic oscillator model.

\section{Introduction}

Many chemical models are governed by difference as well as differential equations. As compared to the continuous model, discrete models designated by difference equations are better explored in recent years. Mathematical models of chemistry, physics, physiology, psychology, ecology, engineering, and social sciences have given birth to major areas of research during the last few decades. For instance, Edeki et al. [1] have explored the numerical solution of the following nonlinear biochemical model by using the hybrid technique:

$$
\begin{gathered}
\eta \frac{\mathrm{d} x}{\mathrm{~d} t}=y-\alpha x-x y, \\
\frac{\mathrm{d} y}{\mathrm{~d} t}=-y+(\alpha-\beta),
\end{gathered}
$$

where $x$ and $y$ are the substrate concentrations at time $t$ and $\eta, \alpha$, and $\beta$ are the dimensionless parameters. Zafar et al. [2] have investigated the equilibria and convergence analysis of the following nonlinear biochemical reaction networks:

$$
\begin{aligned}
& \frac{\mathrm{d} x}{\mathrm{~d} t}=x+(\beta-\alpha)+x y, \\
& \frac{\mathrm{d} y}{\mathrm{~d} t}=\frac{1}{\sigma}(x-\beta y-x y),
\end{aligned}
$$

where $x$ is the concentration of the substrate, $y$ is the intermediate complex, and the parameters $\sigma, \alpha$, and $\beta$ are the dimensionless parameters. Inspired from the aforementioned studies, the goal of this paper is to investigate the bifurcation analysis of a glycolytic oscillator model:

$$
\begin{aligned}
& x_{n+1}=x_{n}+h\left(\alpha-\beta x_{n}-x_{n} y_{n}^{2}\right), \\
& y_{n+1}=y_{n}+h\left(\beta x_{n}+x_{n} y_{n}^{2}-y_{n}\right),
\end{aligned}
$$

which is the discrete analogue of the following continuoustime model, by Euler's forward formula:

$$
\begin{aligned}
& \frac{\mathrm{d} x}{\mathrm{~d} t}=\alpha-\beta x-x y^{2}, \\
& \frac{\mathrm{d} y}{\mathrm{~d} t}=\beta x+x y^{2}-y,
\end{aligned}
$$


where $x$ and $y$, respectively, denote fructose-6-phosphate and adenosine diphosphate and $\alpha$ and $\beta$ are the positive constants. For more detailed background and mathematical modelling of the glycolytic oscillator model (4), the reader is referred to [3-7]. More specifically, the main finding in this article is as follows:

(1) Study of the dynamics about $P_{x y}^{+}\left(\left(\alpha / \beta+\alpha^{2}\right), \alpha\right)$ of model (3)

(2) Existence of possible bifurcation about $P_{x y}^{+}\left(\left(\alpha / \beta+\alpha^{2}\right), \alpha\right)$

(3) To investigate, for the model under consideration, that no other bifurcation exists except the Neimark-Sacker bifurcation

(4) Verification of theoretical results numerically

The rest of the article is organized as follows: Section 2 is about the existence of a positive fixed point in $\mathbb{R}_{+}^{2}$ and the corresponding linearized form of the glycolytic oscillator model (3). Local dynamics about $P_{x y}^{+}\left(\left(\alpha / \beta+\alpha^{2}\right), \alpha\right)$ of (3) is investigated in Section 3. Existence of bifurcation about $P_{x y}^{+}\left(\left(\alpha / \beta+\alpha^{2}\right), \alpha\right)$ is investigated in Section 4, whereas detailed Neimark-Sacker bifurcation analysis is given in Section 5. Simulations are given in Section 6. The conclusion of the paper is given in Section 7.

\section{Existence of Positive Equilibrium Point and Linearized Form of Model (3)}

The existence of a positive fixed point in $\mathbb{R}_{+}^{2}$ and the corresponding linearized form of the model are explored in this section. Specifically, the existence result about the positive fixed point can be stated as the following lemma.

Lemma 1. For all $\alpha$ and $\beta, P_{x y}^{+}\left(\left(\alpha / \beta+\alpha^{2}\right), \alpha\right)$ is the unique positive equilibrium point of model (3).

Hereafter, about $P_{x y}(x, y)$, the linearized form of model (3) is constructed. For the corresponding linearized form of (3), one has the following map:

$$
\left(\Psi_{1}, \Psi_{2}\right) \mapsto\left(x_{n+1}, y_{n+1}\right),
$$

where

$$
\begin{gathered}
\Psi_{1}=x_{n}+h\left(\alpha-\beta x_{n}-x_{n} y_{n}^{2}\right), \\
\Psi_{2}=y_{n}+h\left(\beta x_{n}+x_{n} y_{n}^{2}-y_{n}\right) . \\
\left.J\right|_{P_{x y}(x, y)} \text { about } P_{x y}(x, y) \text { under (5) is } \\
\left.J\right|_{P_{x y}(x, y)}=\left(\begin{array}{cc}
1-h\left(\beta+y^{2}\right) & -2 x y h \\
h\left(\beta+y^{2}\right) & 1-h+2 h x y
\end{array}\right) .
\end{gathered}
$$

\section{Local Dynamics about $P_{x y}^{+}\left(\left(\alpha / \beta+\alpha^{2}\right), \alpha\right)$ of Model (3)}

The local dynamics of the glycolytic oscillator model (3) is explored by utilizing the linearization method. $\left.J\right|_{P_{x y}^{+}\left(\left(\alpha / \beta+\alpha^{2}\right), \alpha\right)}$ about $P_{x y}^{+}\left(\left(\alpha / \beta+\alpha^{2}\right), \alpha\right)$ is
$\left.J\right|_{P_{x y}^{+}\left(\left(\alpha / \beta+\alpha^{2}\right), \alpha\right)}=\left(\begin{array}{cc}1-h\left(\beta+\alpha^{2}\right) & -\frac{2 \alpha^{2} h}{\alpha^{2}+\beta} \\ h\left(\beta+\alpha^{2}\right) & \frac{\left(\alpha^{2}+\beta\right)(1-h)+2 h \alpha^{2}}{\alpha^{2}+\beta}\end{array}\right)$.

The characteristic equation of $\left.J\right|_{P_{x y}^{+}\left(\left(\alpha / \beta+\alpha^{2}\right), \alpha\right)}$ about $P_{x y}^{+}\left(\left(\alpha / \beta+\alpha^{2}\right), \alpha\right)$ is

$$
\lambda^{2}-p \lambda+q=0
$$

where

$$
\begin{aligned}
& p=2-h\left(\beta+\alpha^{2}+1\right)+\frac{2 h \alpha^{2}}{\alpha^{2}+\beta}, \\
& q=(1-h)\left(1-h\left(\alpha^{2}+\beta\right)\right)+\frac{2 h \alpha^{2}}{\alpha^{2}+\beta} .
\end{aligned}
$$

And, the eigenvalues of $\left.J\right|_{P_{x y}^{+}\left(\left(\alpha / \beta+\alpha^{2}\right), \alpha\right)}$ about $P_{x y}^{+}((\alpha / \beta+$ $\left.\left.\alpha^{2}\right), \alpha\right)$ are

$$
\lambda_{1,2}=\frac{p \pm \sqrt{\Delta}}{2},
$$

where

$$
\begin{aligned}
\Delta= & p^{2}-4 q \\
= & \left(2-h\left(\beta+\alpha^{2}+1\right)+\frac{2 h \alpha^{2}}{\alpha^{2}+\beta}\right)^{2}-4\left((1-h)\left(1-h\left(\beta+\alpha^{2}\right)\right)\right. \\
& \left.+\frac{2 h \alpha^{2}}{\alpha^{2}+\beta}\right) .
\end{aligned}
$$

Now, in the following two lemmas, local dynamics about $P_{x y}^{+}\left(\left(\alpha / \beta+\alpha^{2}\right), \alpha\right)$ for the model under consideration is studied.

Lemma 2. If $\Delta=\left(2-h\left(\beta+\alpha^{2}+1\right)+\left(2 h \alpha^{2} / \alpha^{2}+\beta\right)\right)^{2}-$ $4\left((1-h)\left(1-h\left(\alpha^{2}+\beta\right)\right)+\left(2 h \alpha^{2} / \alpha^{2}+\beta\right)\right)<0$, then for $P_{x y}^{+}\left(\left(\alpha / \beta+\alpha^{2}\right), \alpha\right)$, the following holds:

(i) $P_{x y}^{+}\left(\left(\alpha / \beta+\alpha^{2}\right), \alpha\right)$ is a stable focus if

$$
h<1+\frac{\beta-\alpha^{2}}{\left(\beta+\alpha^{2}\right)^{2}} ;
$$

(ii) $P_{x y}^{+}\left(\left(\alpha / \beta+\alpha^{2}\right), \alpha\right)$ is an unstable focus if

$$
h>1+\frac{\beta-\alpha^{2}}{\left(\beta+\alpha^{2}\right)^{2}}
$$

(iii) $P_{x y}^{+}\left(\left(\alpha / \beta+\alpha^{2}\right), \alpha\right)$ is a nonhyperbolic if

$$
h=1+\frac{\beta-\alpha^{2}}{\left(\beta+\alpha^{2}\right)^{2}} .
$$


Lemma 3. If $\Delta=\left(2-h\left(\beta+\alpha^{2}+1\right)+\left(2 h \alpha^{2} / \alpha^{2}+\right.\right.$ $\beta))^{2}-4\left((1-h)\left(1-h\left(\alpha^{2}+\beta\right)\right)+\left(2 h \alpha^{2} / \alpha^{2}+\beta /\right)\right) \geq 0$, then (i) $P_{x y}^{+}\left(\left(\alpha / \beta+\alpha^{2}\right), \alpha\right)$ is a stable node if for $P_{x y}^{+}\left(\left(\alpha / \beta+\alpha^{2}\right), \alpha\right)$, the following holds:

$$
\begin{aligned}
h>\max \left\{\frac{2\left(1+\beta+\alpha^{2}\right)\left(\alpha^{2}+\beta\right)-4 \alpha^{2}-\sqrt{\left(4 \alpha^{2}-2\left(1+\beta+\alpha^{2}\right)\left(\beta+\alpha^{2}\right)\right)^{2}-16\left(\beta+\alpha^{2}\right)^{3}}}{2\left(\alpha^{2}+\beta\right)^{2}},\right. \\
\\
\left.\frac{2\left(1+\beta+\alpha^{2}\right)\left(\alpha^{2}+\beta\right)-4 \alpha^{2}+\sqrt{\left(4 \alpha^{2}-2\left(1+\beta+\alpha^{2}\right)\left(\beta+\alpha^{2}\right)\right)^{2}-16\left(\beta+\alpha^{2}\right)^{3}}}{2\left(\beta+\alpha^{2}\right)^{2}}\right\} ;
\end{aligned}
$$

(ii) $P_{x y}^{+}\left(\left(\alpha / \beta+\alpha^{2}\right), \alpha\right)$ is an unstable node if

$$
\begin{aligned}
h<\min \{ & \frac{2\left(1+\beta+\alpha^{2}\right)\left(\alpha^{2}+\beta\right)-4 \alpha^{2}-\sqrt{\left(4 \alpha^{2}-2\left(1+\beta+\alpha^{2}\right)\left(\beta+\alpha^{2}\right)\right)^{2}-16\left(\beta+\alpha^{2}\right)^{3}}}{2\left(\alpha^{2}+\beta\right)^{2}}, \\
& \left.\frac{2\left(1+\beta+\alpha^{2}\right)\left(\alpha^{2}+\beta\right)-4 \alpha^{2}+\sqrt{\left(4 \alpha^{2}-2\left(1+\beta+\alpha^{2}\right)\left(\beta+\alpha^{2}\right)\right)^{2}-16\left(\beta+\alpha^{2}\right)^{3}}}{2\left(\alpha^{2}+\beta\right)^{2}}\right\} ;
\end{aligned}
$$

(iii) $P_{x y}^{+}\left(\left(\alpha / \beta+\alpha^{2}\right), \alpha\right)$ is a nonhyperbolic if

$$
h=\frac{2\left(1+\beta+\alpha^{2}\right)\left(\alpha^{2}+\beta\right)-4 \alpha^{2}-\sqrt{\left(4 \alpha^{2}-2\left(1+\beta+\alpha^{2}\right)\left(\beta+\alpha^{2}\right)\right)^{2}-16\left(\beta+\alpha^{2}\right)^{3}}}{2\left(\beta+\alpha^{2}\right)^{2}},
$$

or

$$
h=\frac{2\left(1+\beta+\alpha^{2}\right)\left(\alpha^{2}+\beta\right)-4 \alpha^{2}+\sqrt{\left(4 \alpha^{2}-2\left(1+\beta+\alpha^{2}\right)\left(\alpha^{2}+\beta\right)\right)^{2}-16\left(\alpha^{2}+\beta\right)^{3}}}{2\left(\alpha^{2}+\beta\right)^{2}} .
$$

Now, the existence of bifurcation about $P_{x y}^{+}\left(\left(\alpha / \beta+\alpha^{2}\right), \alpha\right)$ is explored based on the above theoretical results.

\section{Existence of}

Bifurcations about $P_{x y}^{+}\left(\left(\alpha / \beta+\alpha^{2}\right), \alpha\right)$

(i) From Lemma 2, one can obtain that if (15) holds, then

$\left|\lambda_{1,2}\right|=\left|\frac{2-h\left(\beta+\alpha^{2}+1\right)+2 h \alpha^{2} /\left(\alpha^{2}+\beta\right) \pm \iota \sqrt{4\left((1-h)\left(1-h\left(\alpha^{2}+\beta\right)\right)+\left(2 h \alpha^{2} / \alpha^{2}+\beta\right)\right)-\left(2-h\left(\beta+\alpha^{2}+1\right)+\left(2 h \alpha^{2} / \alpha^{2}+\beta\right)\right)^{2}}}{2}\right|=1$

Equation (20) implies that model (3) undergoes Neimark-Sacker bifurcation if $(\alpha, \beta, h)$ are in the following set:

$$
\mathrm{HB}_{P_{x y}^{+}\left(\left(\alpha / \beta+\alpha^{2}\right), \alpha\right)}=\left\{(\alpha, \beta, h): h=1+\frac{\beta-\alpha^{2}}{\left(\beta+\alpha^{2}\right)^{2}}\right\} .
$$


(ii) There does not exist period-doubling bifurcation as eigenvalues of $\left.\quad J\right|_{P_{x,}^{+}\left(\left(\alpha / \beta+\alpha^{2}\right), \alpha\right)}$ about $P_{x y}^{+}\left(\left(\alpha / \beta+\alpha^{2}\right), \alpha\right)$ are neither -1 nor 1 if (18) or (19) holds.

\section{Neimark-Sacker}

Bifurcation about $P_{x y}^{+}\left(\left(\alpha / \beta+\alpha^{2}\right), \alpha\right)$

Hereafter, by using bifurcation theory $[8,9]$, the detailed Neimark-Sacker bifurcation about $P_{x y}^{+}\left(\left(\alpha / \beta+\alpha^{2}\right), \alpha\right)$ is

$$
\left.J\right|_{P_{x y}^{+}\left(\left(\alpha / \beta+\alpha^{2}\right), \alpha\right)}=\left(\begin{array}{cc}
1-\left(h^{*}+\varepsilon\right)\left(\beta+\alpha^{2}\right) & -\frac{2\left(h^{*}+\varepsilon\right) \alpha^{2}}{\beta+\alpha^{2}} \\
\left(h^{*}+\varepsilon\right)\left(\beta+\alpha^{2}\right) & \frac{\left(\beta+\alpha^{2}\right)\left(1-\left(h^{*}+\varepsilon\right)\right)+2\left(h^{*}+\varepsilon\right) \alpha^{2}}{\alpha^{2}+\beta}
\end{array}\right) .
$$

The characteristic equation of $\left.J\right|_{P_{x y}^{+}\left(\left(\alpha / \beta+\alpha^{2}\right), \alpha\right)}$ about
$\left(\left(\alpha / \beta+\alpha^{2}\right), \alpha\right)$ is $P_{x y}^{+}\left(\left(\alpha / \beta+\alpha^{2}\right), \alpha\right)$ is

$$
\lambda^{2}-p(\varepsilon) \lambda+q(\varepsilon)=0
$$

where

$$
\begin{aligned}
& p(\varepsilon)=2-\left(h^{*}+\varepsilon\right)\left(\beta+\alpha^{2}+1\right)+\frac{2\left(h^{*}+\varepsilon\right) \alpha^{2}}{\alpha^{2}+\beta}, \\
& q(\varepsilon)=\left(1-\left(h^{*}+\varepsilon\right)\right)\left(1-\left(h^{*}+\varepsilon\right)\left(\beta+\alpha^{2}\right)\right)+\frac{2\left(h^{*}+\varepsilon\right) \alpha^{2}}{\alpha^{2}+\beta} .
\end{aligned}
$$

From (24), one gets

$$
\begin{aligned}
\lambda_{1,2}= & \frac{p(\varepsilon) \pm \iota \sqrt{4 q(\varepsilon)-p^{2}(\varepsilon)}}{2} \\
= & \frac{2-\left(h^{*}+\varepsilon\right)\left(\beta+\alpha^{2}+1\right)+\left(\left(2\left(h^{*}+\varepsilon\right) \alpha^{2}\right) / \alpha^{2}+\beta\right)}{2} \\
& \pm \frac{\iota}{2} \sqrt{\Delta^{*}},
\end{aligned}
$$

where

$$
\begin{gathered}
\Delta^{*}=4\left(\left(1-\left(h^{*}+\varepsilon\right)\right)\left(1-\left(h^{*}+\varepsilon\right)\left(\alpha^{2}+\beta\right)\right)+\frac{2\left(h^{*}+\varepsilon\right) \alpha^{2}}{\alpha^{2}+\beta}\right) \\
-\left(2-\left(h^{*}+\varepsilon\right)\left(\beta+\alpha^{2}+1\right)+\frac{2\left(h^{*}+\varepsilon\right) \alpha^{2}}{\alpha^{2}+\beta}\right)^{2} \\
\left.\frac{\mathrm{d}\left|\lambda_{1,2}\right|}{\mathrm{d} \varepsilon}\right|_{\varepsilon=0}=\frac{\beta-\alpha^{2}+\left(\alpha^{2}+\beta\right)^{2}}{2\left(\beta+\alpha^{2}\right)}>0 .
\end{gathered}
$$

Additionally, it is required that $\lambda_{1,2}^{\ell} \neq 1, \ell=1, \ldots, 4$, which corresponds to $p(0) \neq 1,2,0,-1$ and so it is true by calculation. Now, if $u_{n}=x_{n}-x^{*}, v_{n}=y_{n}-y^{*}$, then explored if $h$ goes through $\operatorname{HB}_{P_{x y}^{+}}\left(\left(\alpha / \beta+\alpha^{2}\right), \alpha\right)$. Now, if $h$ varies in a small nbhd of $h^{*}$, that is, $h=h^{*}+\varepsilon$ with $\varepsilon \ll 1$, then model (3) becomes

$$
\begin{aligned}
& x_{n+1}=x_{n}+\left(h^{\star}+\varepsilon\right)\left(\alpha-\beta x_{n}-x_{n} y_{n}^{2}\right), \\
& y_{n+1}=y_{n}+\left(h^{\star}+\varepsilon\right)\left(\beta x_{n}+x_{n} y_{n}^{2}-y_{n}\right) .
\end{aligned}
$$

$\left.J\right|_{P_{x y}^{+}\left(\left(\alpha / \beta+\alpha^{2}\right), \alpha\right)}$ about $P_{x y}^{+}\left(\left(\alpha / \beta+\alpha^{2}\right), \alpha\right)$ of $(22)$ is

$P_{x y}^{+}\left(\left(\alpha / \beta+\alpha^{2}\right), \alpha\right)$ of the glycolytic oscillator model (3) transforms into $O(0,0)$. So,

$$
\begin{aligned}
u_{n+1}= & u_{n}+\left(h^{*}+\varepsilon\right)\left(\alpha-\beta\left(u_{n}+x^{*}\right)-\left(u_{n}+x^{*}\right)\left(v_{n}+y^{*}\right)^{2}\right), \\
v_{n+1}= & v_{n}+\left(h^{*}+\varepsilon\right)\left(\beta\left(u_{n}+x^{*}\right)+\left(u_{n}+x^{*}\right)\left(v_{n}+y^{*}\right)^{2}\right. \\
& \left.-\left(v_{n}+y^{*}\right)\right),
\end{aligned}
$$

where $x^{*}=\left(\alpha / \beta+\alpha^{2}\right)$ and $y^{*}=\alpha$. Hereafter, the normal form of (29) is studied if $\varepsilon=0$. From (29), one gets

$$
\begin{aligned}
& u_{n+1}=a_{11} u_{n}+a_{12} v_{n}+a_{13} u_{n} v_{n}+a_{14} v_{n}^{2}+a_{15} u_{n} v_{n}^{2}, \\
& v_{n+1}=a_{21} u_{n}+a_{22} v_{n}+a_{23} u_{n} v_{n}+a_{24} v_{n}^{2}+a_{25} u_{n} v_{n}^{2},
\end{aligned}
$$

where

$$
\begin{aligned}
& a_{11}=1-h^{*}\left(\beta+y^{*^{2}}\right), \\
& a_{12}=-2 h^{*} x^{*} y^{*}, \\
& a_{13}=-2 h^{*} y^{*}, \\
& a_{14}=-h^{*} x^{*}, \\
& a_{15}=-h^{*}, \\
& a_{21}=h^{*}\left(\beta+y^{*^{2}}\right), \\
& a_{22}=1+h^{*}\left(2 x^{*} y^{*}-1\right), \\
& a_{23}=2 h^{*} y^{*}, \\
& a_{24}=h^{*} x^{*}, \\
& a_{25}=h^{*} .
\end{aligned}
$$

Now, the matrix $T$ is obtained that puts the linear part of (30) into a conoidal form:

$$
T:=\left(\begin{array}{cc}
a_{12} & 0 \\
\eta-a_{11} & -\zeta
\end{array}\right),
$$

where 


$$
\begin{aligned}
& \eta=\frac{2-h^{*}\left(\beta+\alpha^{2}+1\right)+\left(2 h^{*} \alpha^{2} / \alpha^{2}+\beta\right)}{2}, \\
& \zeta=\frac{1}{2} \sqrt{\Delta^{*}},
\end{aligned}
$$

and $\Delta^{*}$ is depicted in (27). Hence, (30) then implies that

$$
\begin{aligned}
& X_{n+1}=\eta X_{n}-\zeta Y_{n}+\bar{P}, \\
& Y_{n+1}=\zeta X_{n}+\eta Y_{n}+\bar{Q},
\end{aligned}
$$

where

$$
\begin{aligned}
\bar{P}\left(X_{n}, Y_{n}\right)= & l_{11} X_{n}^{2}+l_{12} X_{n} Y_{n}+l_{13} Y_{n}^{2}+l_{14} X_{n}^{3}+l_{15} X_{n} Y_{n}^{2} \\
& +l_{16} Y_{n} X_{n}^{2}, \\
\bar{Q}\left(X_{n}, Y_{n}\right)= & l_{21} X_{n}^{2}+l_{22} X_{n} Y_{n}+l_{23} Y_{n}^{2}+l_{24} X_{n}^{3}+l_{25} X_{n} Y_{n}^{2} \\
& +l_{26} Y_{n} X_{n}^{2},
\end{aligned}
$$$$
l_{11}=\frac{\eta-a_{11}}{a_{12}}\left[a_{12} a_{13}+a_{14}\left(\eta-a_{11}\right)\right],
$$$$
l_{12}=-\frac{\zeta}{a_{12}}\left[a_{12} a_{13}+2 a_{14}\left(\eta-a_{11}\right)\right]
$$$$
l_{13}=\frac{a_{14}}{a_{12}} \zeta^{2},
$$$$
l_{14}=a_{15}\left(\eta-a_{11}\right)^{2},
$$$$
l_{15}=a_{15} \zeta^{2} \text {, }
$$$$
l_{16}=-2 a_{15} \zeta\left(\eta-a_{11}\right) \text {, }
$$$$
l_{21}=\frac{\eta-a_{11}}{\zeta a_{12}}\left[\left(\eta-a_{11}\right)\left(a_{12}\left(a_{13}-a_{24}\right)+a_{14}\left(\eta-a_{11}\right)\right)\right.
$$$$
\left.-a_{12}^{2} a_{23}\right]
$$$$
l_{22}=\frac{\eta-a_{11}}{a_{12}}\left[\left(\eta-a_{11}\right)\left(2 a_{12} a_{24}-2 a_{14}\right)-a_{12} a_{13}\right]-a_{12} a_{23},
$$$$
l_{23}=\frac{\zeta}{a_{12}}\left[a_{14}\left(\eta-a_{11}\right)-a_{12} a_{24}\right] \text {, }
$$$$
l_{24}=\frac{\left(\eta-a_{11}\right)^{2}}{\zeta}\left[a_{15}\left(\eta-a_{11}\right)-a_{12} a_{25}\right],
$$$$
l_{25}=\zeta\left[a_{15}\left(\eta-a_{11}\right)-a_{12} a_{25}\right],
$$$$
l_{26}=\left(\eta-a_{11}\right)\left[2 a_{12} a_{25}-2 a_{15}\left(\eta-a_{11}\right)^{2}\right] \text {, }
$$

by

$$
\left(\begin{array}{c}
u_{n} \\
v_{n}
\end{array}\right):=\left(\begin{array}{cc}
a_{12} & 0 \\
\eta-a_{11} & -\zeta
\end{array}\right)\left(\begin{array}{c}
X_{n} \\
Y_{n}
\end{array}\right)
$$

Also,

$$
\begin{aligned}
\left.\bar{P}_{X_{n} X_{n}}\right|_{O} & =2 l_{11}, \\
\left.\bar{P}_{X_{n} Y_{n}}\right|_{O} & =l_{12}, \\
\left.\bar{P}_{Y_{n} Y_{n}}\right|_{O} & =2 l_{13}, \\
\left.\bar{P}_{X_{n} X_{n} X_{n}}\right|_{O} & =6 l_{14}, \\
\left.\bar{P}_{X_{n} X_{n} Y_{n}}\right|_{O} & =2 l_{16}, \\
\left.\bar{P}_{X_{n} Y_{n} Y_{n}}\right|_{O} & =2 l_{15}, \\
\left.\bar{P}_{Y_{n} Y_{n} Y_{n}}\right|_{O} & =0, \\
\left.\bar{Q}_{X_{n} X_{n}}\right|_{O} & =2 l_{21}, \\
\left.\bar{Q}_{X_{n} Y_{n}}\right|_{O} & =l_{22}, \\
\left.\bar{Q}_{Y_{n} Y_{n}}\right|_{O} & =2 l_{23}, \\
\left.\bar{Q}_{X_{n} X_{n} X_{n}}\right|_{O} & =6 l_{24}, \\
\left.\bar{Q}_{X_{n} X_{n} Y_{n}}\right|_{O} & =2 l_{26}, \\
\left.\bar{Q}_{X_{n} Y_{n} Y_{n}}\right|_{O} & =2 l_{25}, \\
\left.\bar{Q}_{Y_{n} Y_{n} Y_{n}}\right|_{O} & =0,
\end{aligned}
$$

It is noted that the following relation should be nonzero in order for (34) to undergo the Neimark-Sacker bifurcation (see [8-16]):

$$
\Omega=-\operatorname{Real}\left[\frac{(1-2 \bar{\lambda}) \bar{\lambda}^{2}}{1-\lambda} \xi_{11} \xi_{20}\right]-\frac{1}{2}\left\|\xi_{11}\right\|^{2}-\left\|\xi_{02}\right\|^{2}+\operatorname{Real}\left(\bar{\lambda} \xi_{21}\right),
$$

where

$$
\begin{aligned}
\xi_{02}= & \left.\frac{1}{8}\left[\bar{P}_{X_{n} X_{n}}-\bar{P}_{Y_{n} Y_{n}}+2 \bar{Q}_{X_{n} Y_{n}}+\iota\left(\bar{Q}_{X_{n} X_{n}}-\bar{Q}_{Y_{n} Y_{n}}+2 \bar{P}_{X_{n} Y_{n}}\right)\right]\right|_{O}, \\
\xi_{11}= & \left.\frac{1}{4}\left[\bar{P}_{X_{n} X_{n}}+\bar{P}_{Y_{n} Y_{n}}+\iota\left(\bar{Q}_{X_{n} X_{n}}+\bar{Q}_{Y_{n} Y_{n}}\right)\right]\right|_{O} \\
\xi_{20}= & \left.\frac{1}{8}\left[\bar{P}_{X_{n} X_{n}}-\bar{P}_{Y_{n} Y_{n}}+2 \bar{Q}_{X_{n} Y_{n}}+\iota\left(\bar{Q}_{X_{n} X_{n}}-\bar{Q}_{Y_{n} Y_{n}}-2 \bar{P}_{X_{n} Y_{n}}\right)\right]\right|_{O} \\
\xi_{21}= & \frac{1}{16}\left[\bar{P}_{X_{n} X_{n} X_{n}}+\bar{P}_{X_{n} Y_{n} Y_{n}}+\bar{Q}_{X_{n} X_{n} Y_{n}}+\bar{Q}_{Y_{n} Y_{n} Y_{n}}\right. \\
& \left.+\iota\left(\bar{Q}_{X_{n} X_{n} X_{n}}+\bar{Q}_{X_{n} Y_{n} Y_{n}}-\bar{P}_{X_{n} X_{n} Y_{n}}-P_{Y_{n} Y_{n} Y_{n}}\right)\right]\left.\right|_{O} .
\end{aligned}
$$

After manipulation, one gets 


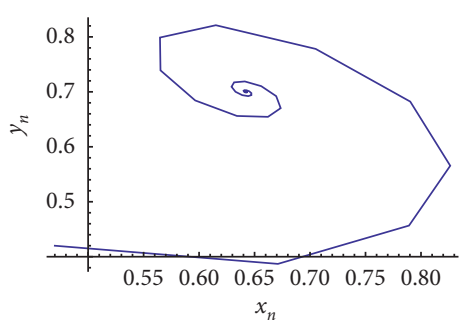

(a)

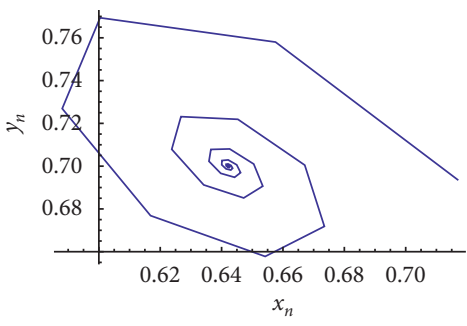

(d)

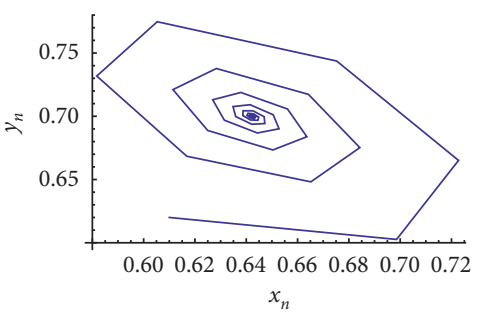

(g)

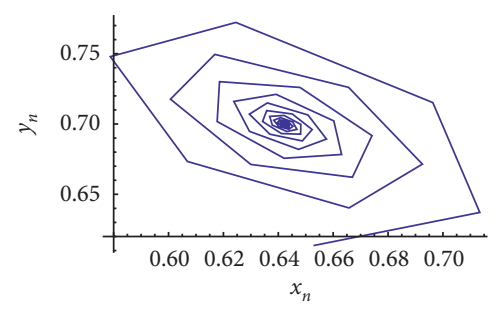

(j)

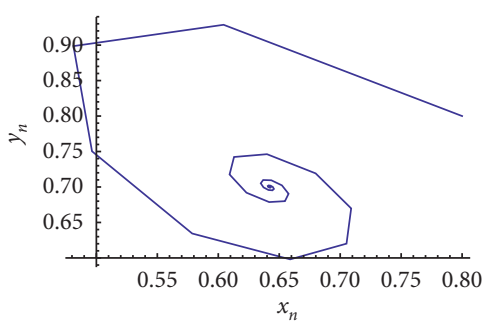

(b)

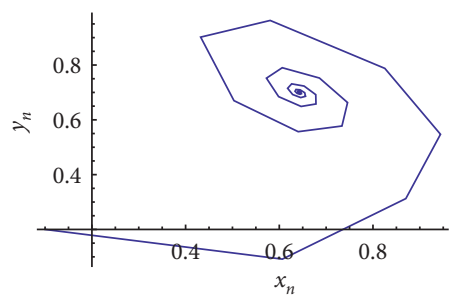

(e)

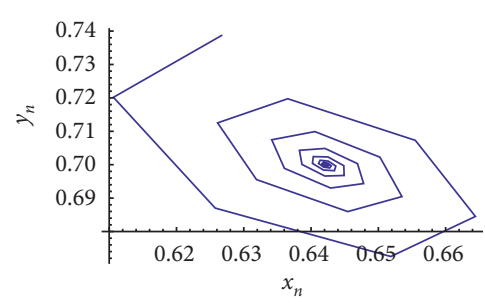

(h)

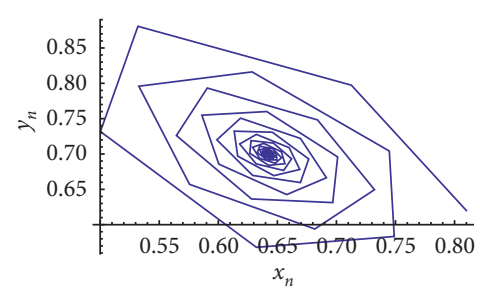

(k)

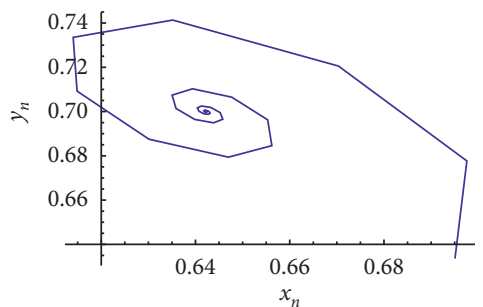

(c)

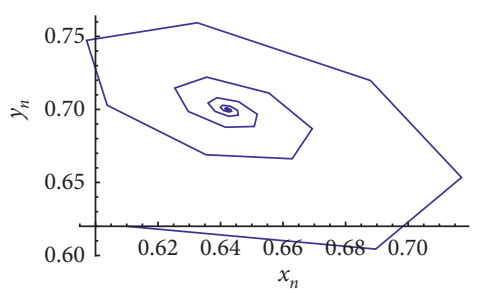

(f)

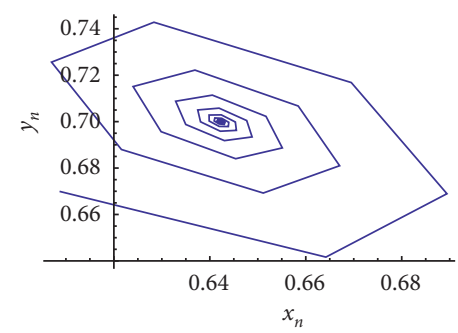

(i)

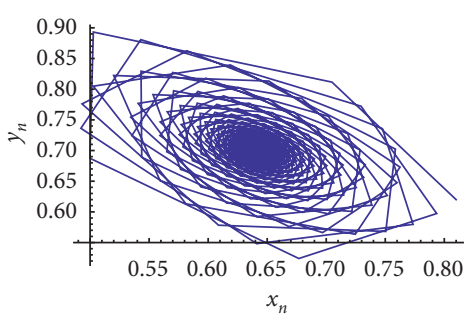

(l)

Figure 1: Trajectories of model (3). (a) $h=0.6$ with $(0.47 ; 0.42)$, (b) $h=0.67$ with $(0.8 ; 0.8)$, (c) $h=0.7$ with $(0.38 ; 0.38)$, (d) $h=0.79$ with $(0.38$; $0.38)$, (e) $h=0.7965$ with $(0.1 ; 0.2)$, (f) $h=0.8$ with $(0.61 ; 0.62)$, (g) $h=0.89$ with $(0.61 ; 0.62)$, (h) $h=0.894$ with $(0.61 ; 0.62)$, (i) $h=0.9$ with (0.81;0.62), (j) $h=0.97$ with $(0.81 ; 0.62)$, (k) $h=1.0$ with $(0.81 ; 0.62)$, and (l) $h=1.08$ with $(0.81 ; 0.62)$.

$$
\begin{aligned}
& \xi_{02}=\frac{1}{4}\left[l_{11}-l_{13}+l_{22}+\iota\left(l_{21}+l_{12}-l_{23}\right)\right], \\
& \xi_{11}=\frac{1}{2}\left[l_{11}+l_{13}+\iota\left(l_{21}+l_{23}\right)\right], \\
& \xi_{20}=\frac{1}{4}\left[l_{11}-l_{13}+l_{22}+\iota\left(l_{21}-l_{23}-l_{12}\right)\right], \\
& \xi_{21}=\frac{1}{8}\left[3 l_{14}+l_{15}+l_{26}+\iota\left(3 l_{24}+l_{25}-l_{16}\right)\right] .
\end{aligned}
$$

From the analysis and Neimark-Sacker bifurcation conditions discussed in $[8,9]$, one has the following results.

Theorem 1. If (39) holds, then the glycolytic oscillator model (3) undergoes Neimark-Sacker bifurcation about $P_{x y}^{+}((\alpha / \beta+$ $\left.\left.\alpha^{2}\right), \alpha\right)$ as $(\alpha, \beta, h)$ pass through $H B_{P_{\alpha y}^{+}\left(\left(\alpha / \beta+\alpha^{2}\right), \alpha\right)}$. Furthermore, attracting (respectively, repelling) the closed curve bifurcates from $P_{x y}^{+}\left(\left(\alpha / \beta+\alpha^{2}\right), \alpha\right)$ if $\Omega<0$ (respectively, $\left.\Omega>0\right)$.

Remark 1. It is noted here that bifurcation is supercritical (respectively, subcritical) Neimark-Sacker bifurcation if $\Omega<0$ (respectively, $\Omega>0$ ). In the next section, simulations guarantee that (3) undergoes supercritical Neimark-Sacker bifurcation if $(\alpha, \beta, h)$ vary in an nbhd of $P_{x y}^{+}\left(\left(\alpha / \beta+\alpha^{2}\right), \alpha\right)$.

\section{Numerical Simulations}

Some simulations will be presented for the correctness of the obtained results in this section. For instance, if $\alpha=0.7$ and $\beta=0.6$, then from (15), one gets 


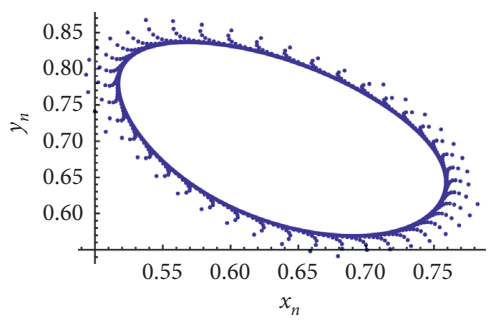

(a)

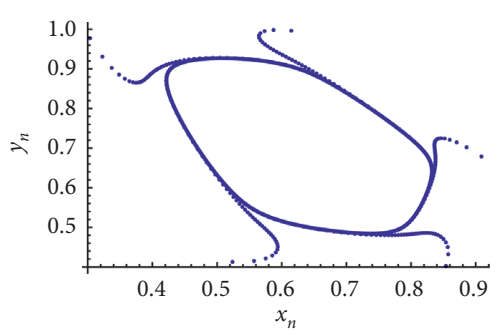

(d)

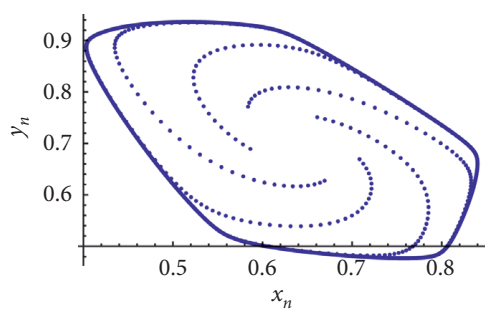

(g)

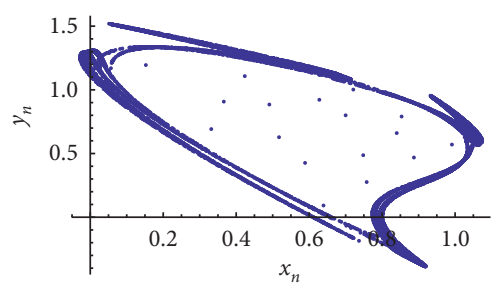

(j)

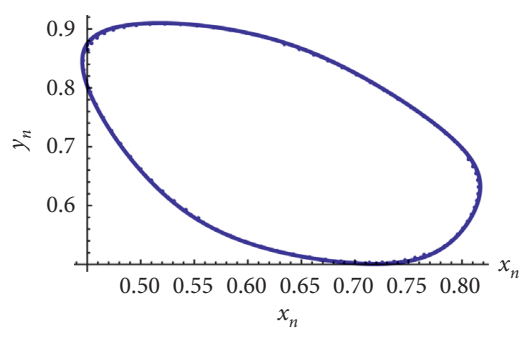

(b)

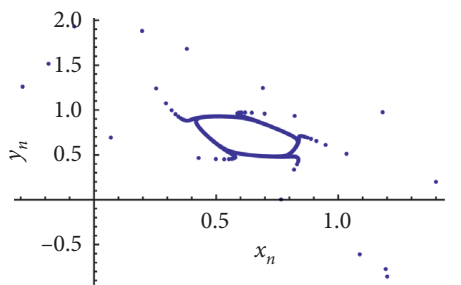

(e)

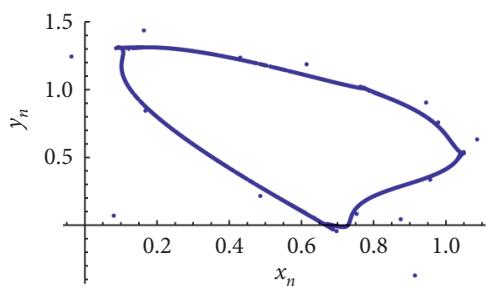

(h)

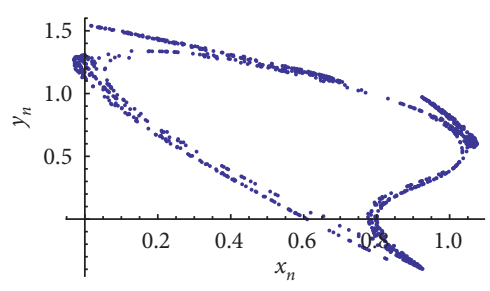

(k)

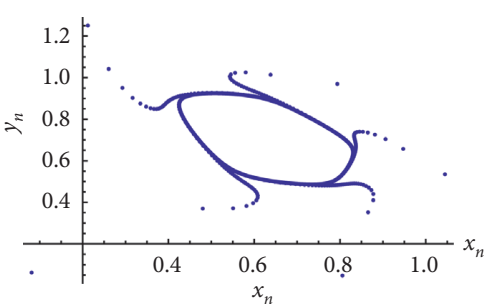

(c)

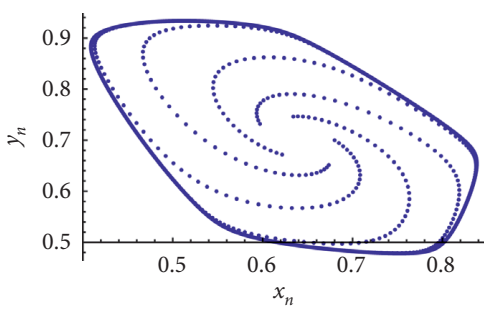

(f)

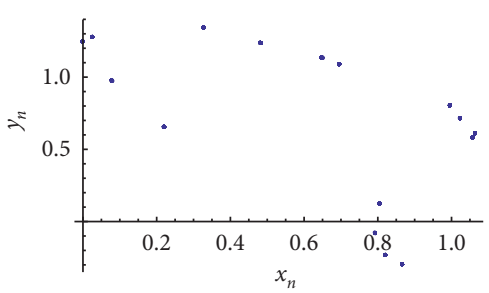

(i)

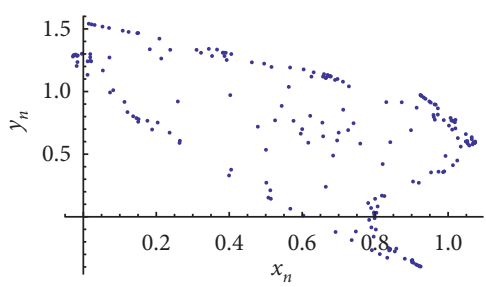

(l)

FiguRe 2: Supercritical Neimark-Sacker bifurcation of model (3). (a) $h=1.1$ with $(0.81 ; 0.62)$, (b) $h=1.11$ with $(0.81 ; 0.62)$, (c) $h=1.113$ with (0.081;0.062), (d) $h=1.11345$ with $(0.01 ; 0.02)$, (e) $h=1.114$ with $(1: 4 ; 0.2)$, (f) $h=1.115$ with $(0.68 ; 0.7),(\mathrm{g}) h=1.1156$ with $(0.68 ; 0.67),(\mathrm{h})$ $h=1: 22$ with (0.08;0.07), (i) $h=1.235$ with $(0.1 ; 0.8),(\mathrm{j}) h=1.245$ with $(0.7 ; 0.8)$, (k) $h=1.247$ with $(0.7 ; 0.9)$, and (l) $h=1.2479$ with $(0.6 ; 0.7)$.

$h=1.0925847992593216$. Theoretically, equilibrium $P_{x y}^{+}\left(\left(\alpha / \beta+\alpha^{2}\right), \alpha\right)$ of (3) is a stable focus if $h<1.0925847992593216$. To see this, if $h=0.6<1.0925847992593216$, then Figure 1(a) implies that $P_{x y}^{+}\left(\left(\alpha / \beta+\alpha^{2}\right), \alpha\right)$ is a stable focus. Similarly, for other values of $h$, if $h<1.0925847992593216$, then $P_{x y}^{+}\left(\left(\alpha / \beta+\alpha^{2}\right), \alpha\right)$ of (3) is a stable focus (see Figures $1(\mathrm{~b})-1(1))$. But, if $h$ goes through 1.0925847992593216, then $P_{x y}^{+}\left(\left(\alpha / \beta+\alpha^{2}\right), \alpha\right)$ becomes unstable, and as a consequence, an attracting closed curve appears. This closed curve indicates that model (3) undergoes a supercritical Neimark-Sacker bifurcation if $(\alpha, \beta, h)$ go through the curve, which is depicted in (21). To see this, if $h=1.1>1.0925847992593216$, then eigenvalues of $\quad P_{x y}^{+}(0.6422018348623854,0.7) \quad$ about $(0.6422018348623854,0.7)$ are

$$
\lambda_{1,2}=0.3449954128440368 \pm 0.9433286759155826 \iota,
$$

and the nongenerate for the existence of Neimark-Sacker bifurcation holds, i.e., $\beta-\alpha^{2}+\left(\alpha^{2}+\beta\right)^{2} / 2\left(\alpha^{2}+\beta\right)=0.5954587155963303>0$. Moreover, after some manipulation from (41), one gets

$$
\begin{aligned}
& \xi_{02}=0.2735375582937717+0.3287376065788703 \iota, \\
& \xi_{11}=0.004623532110091866-0.0021810537860846857 l, \\
& \xi_{20}=0.2735375582937717-0.03107799351733123 \iota, \\
& \xi_{21}=-0.3481094018033289-0.025816775834865524 \iota .
\end{aligned}
$$


TABLE 1: Values of $\Omega$ for $h>1.0925847992593216$.

\begin{tabular}{lr}
\hline Value of $h$ if $h>1.0925847992593216$ & Numerical value of $\Omega$ \\
\hline 1.1 & $-0.32927462274555946<0$ \\
1.11 & $-0.33856187233019613<0$ \\
1.113 & $-0.34138558178129375<0$ \\
1.11345 & $-0.3418106432332544<0$ \\
1.114 & $-0.3423306971220448<0$ \\
1.115 & $-0.343277757130833<0$ \\
1.1156 & $-0.3438469278876272<0$ \\
1.22 & $-0.4541948828452207<0$ \\
1.235 & $-0.472021931523424<0$ \\
1.245 & $-0.48420168645269523<0$ \\
1.247 & $-0.4866663794705075<0$ \\
1.2479 & $-0.48777863891987355<0$ \\
\hline
\end{tabular}

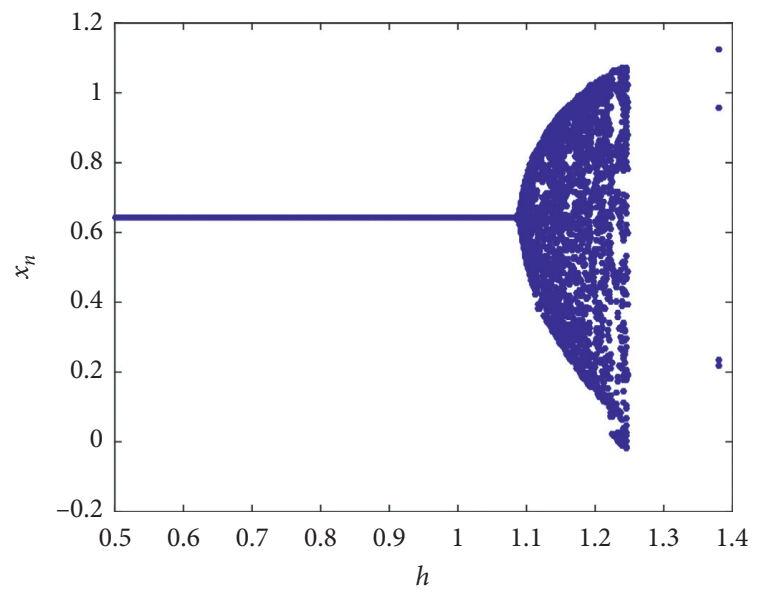

(a)

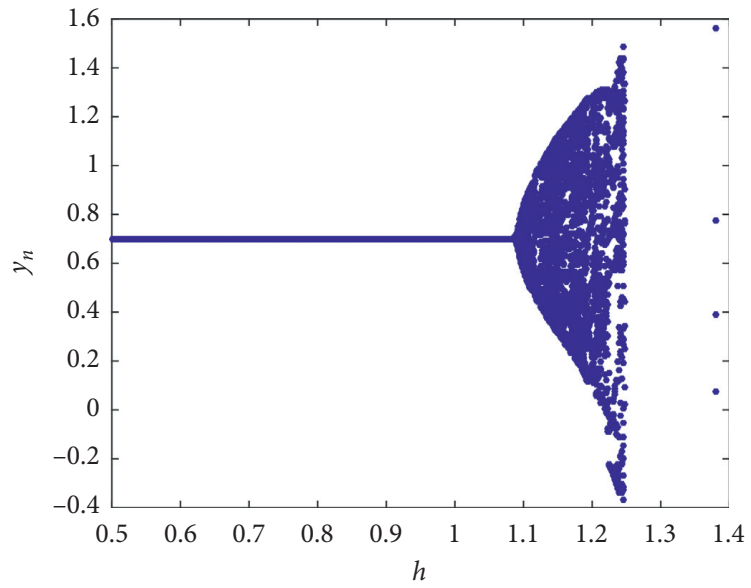

(b)

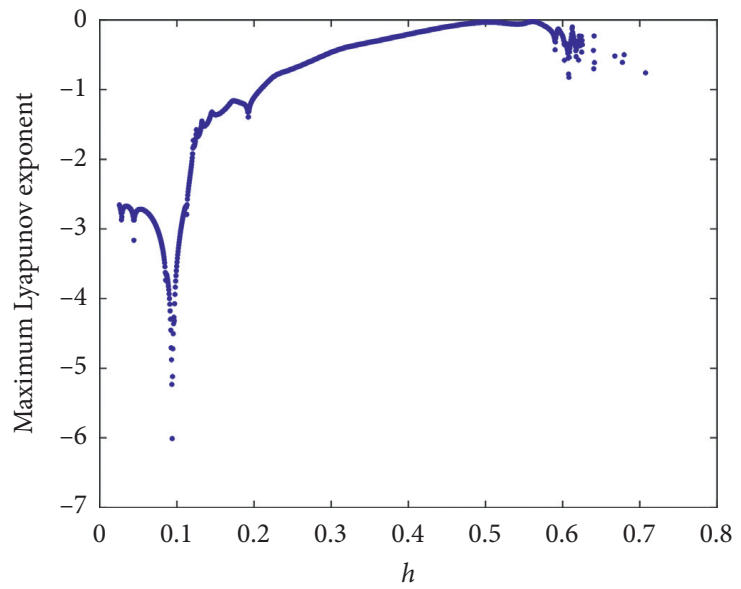

(c)

Figure 3: (a-b) Bifurcation diagram of the model if $h \in[0.5,1.5]$ with the initial condition $(0.47,0.42)$. (c) Maximum Lyapunov exponent corresponding to (a-b).

Using (42) and (43) in (39), one gets $\Omega=-0.32927462274555946<0$. So, if $h=1.1$, model (3) undergoes supercritical Neimark-Sacker bifurcation, and hence a stable curve appears (see Figure 2(a)). In particular, the occurrence of closed curves indicates that model (3) undergoes a supercritical Neimark-Sacker bifurcation, i.e., FGP and ADP coexist with a long time. Also, for the rest of the values of parameters, the values of $\Omega<0$ (see Table 1 ) and corresponding closed curves are presented in Figures 2(b)2(l). Moreover, bifurcation diagrams along with the maximum Lyapunov exponent are plotted in Figure 3. Also, 3D bifurcation diagrams are plotted and drawn in Figure 4. Finally, the topological classification about $P_{x y}^{+}\left(\left(\alpha / \beta+\alpha^{2}\right), \alpha\right)$ of model (3) is presented in Figure 5. 


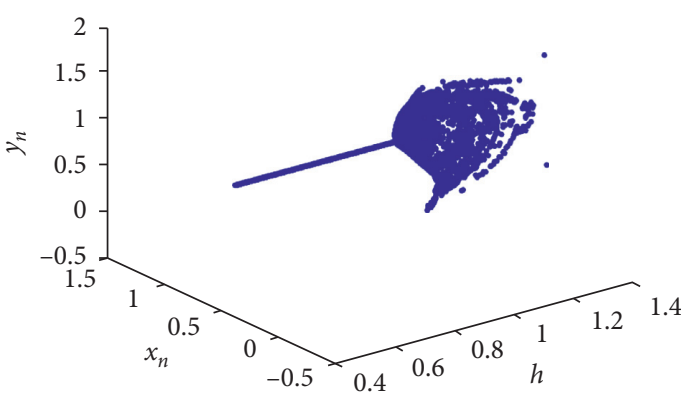

(a)

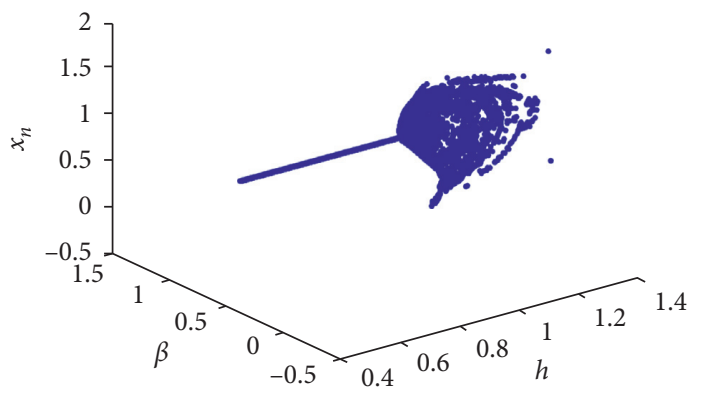

(c)

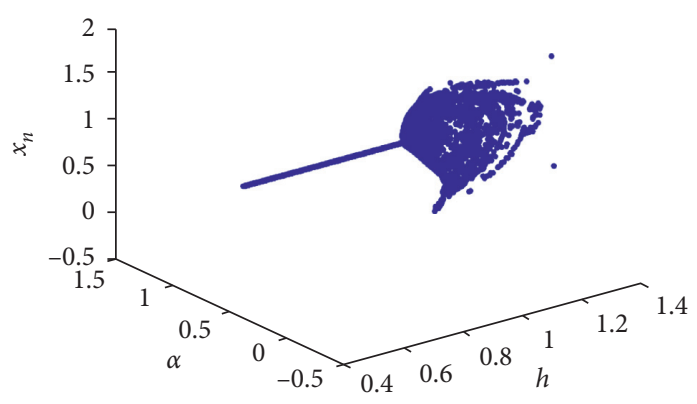

(b)

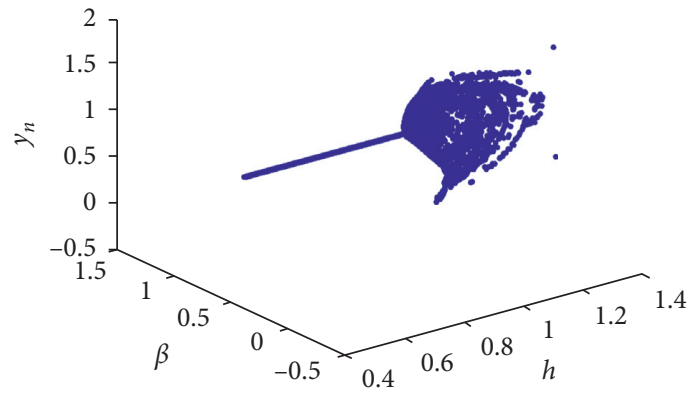

(d)

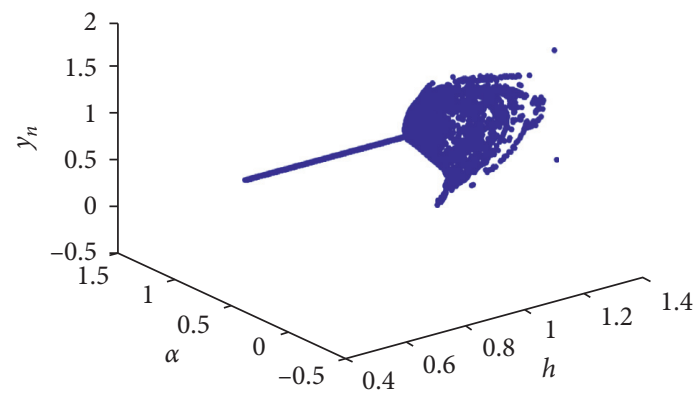

(e)

FIGURE 4: $3 \mathrm{D}$ bifurcation diagrams of model (3).

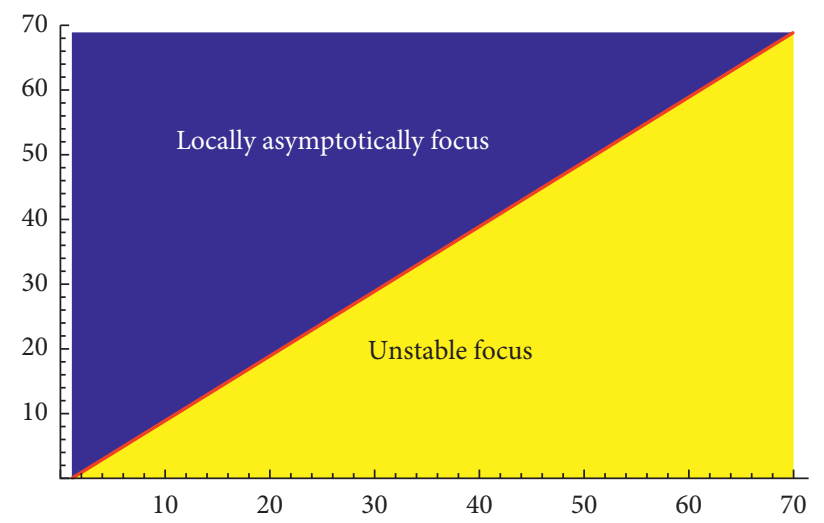

Figure 5: Topological classification about $P_{x y}^{+}\left(\left(\alpha / \beta+\alpha^{2}\right), \alpha\right)$ of model (3). 


\section{Conclusion}

The dynamics and Neimark-Sacker bifurcation of the glycolytic oscillator model in $\mathbb{R}_{+}^{2}$ have been investigated. It has been proved that for all $\alpha$ and $\beta$, model (3) has a positive equilibrium point: $P_{x y}^{+}\left(\left(\alpha / \beta+\alpha^{2}\right), \alpha\right)$. The local dynamics about $P_{x y}^{+}\left(\left(\alpha / \beta+\alpha^{2}\right), \alpha\right)$ has been studied by the method of linearization. It is proved that $P_{x y}^{+}\left(\left(\alpha / \beta+\alpha^{2}\right), \alpha\right)$ is a stable focus if $h<1+\beta-\alpha^{2} /\left(\beta+\alpha^{2}\right)^{2}$, unstable focus if $h>1+\beta-\alpha^{2} /\left(\beta+\alpha^{2}\right)^{2}$, and nonhyperbolic if $h=1+\beta-\alpha^{2} /\left(\beta+\alpha^{2}\right)^{2}$. Further, it is investigated that if $h=1+\beta-\alpha^{2} /\left(\beta+\alpha^{2}\right)^{2}$, then (20) holds which implies that (3) undergoes Neimark-Sacker bifurcation when $(\alpha, \beta, h)$ are located in the set: $\mathrm{HB}_{P_{x y}^{+}\left(\left(\alpha / \beta+\alpha^{2}\right), \alpha\right)}=\left\{(h, \alpha, \beta): h=1+\beta-\alpha^{2} /\left(\beta+\alpha^{2}\right)^{2}\right\}$. Then, Neimark-Sacker bifurcation about $P_{x y}^{+}\left(\left(\alpha / \beta+\alpha^{2}\right), \alpha\right)$ is studied by using bifurcation theory. It is also proved that under certain parametric conditions, $P_{x y}^{+}\left(\left(\alpha / \beta+\alpha^{2}\right), \alpha\right)$ is a stable node, unstable node, and nonhyperbolic. It is also explored that for the model under consideration, no other bifurcation occurs except the Neimark-Sacker bifurcation. Finally, theoretical results are verified numerically.

\section{Data Availability}

All the data utilized in this article have been included, and the sources from where they were adopted are cited accordingly.

\section{Conflicts of Interest}

The author declares that there are no conflicts of interest.

\section{Acknowledgments}

This research by A. Q. Khan was partially supported by the Higher Education Commission of Pakistan.

\section{References}

[1] S. O. Edeki, E. A. Owoloko, A. S. Osheku, A. A. Opanuga, H. I. Okagbue, and G. O. Akinlabi, "Numerical solutions of nonlinear biochemical model using a hybrid numerical-analytical technique," International Journal of Mathematical Analysis, vol. 9, no. 8, pp. 403-416, 2015.

[2] Z. U. A. Zafar, K. Rehan, M. Mushtaq, and M. Rafiq, "Numerical modeling for non-linear biochemical reaction networks," Iranian Journal of Mathematical Chemistry, vol. 8, no. 4, pp. 413-423, 2017.

[3] R. H. Garrett and C. M. Grisham, Biochemistry, Saunder's College Publishing, Philadelphia, PA, USA, 1999.

[4] J. M. Berg, J. L. Tymoczko, and L. Stryer, Biochemistry, Macmillan, New York, NY, USA, 2002.

[5] T. Dandekar, S. Schuster, B. Snel, M. Huynen, and P. Bork, "Pathway alignment: application to the comparative analysis of glycolytic enzymes," Biochemical Journal, vol. 343, no. 1, pp. 115-124, 1999.

[6] A. Boiteux and B. Hess, "Design of glycolysis," Philosophical Transactions of the Royal Society of London. B, Biological Sciences, vol. 293, no. 1063, pp. 5-22, 1981.

[7] L. E. Keshet, Mathematical Models in Bioilogy, Siam, Philadelphia, PA, USA, 1988.
[8] J. Guckenheimer and P. Holmes, Nonlinear Oscillations, Dynamical Systems and Bifurcation of Vector Fields, SpringerVerlag, New York, NY, USA, 1983.

[9] Y. A. Kuznetsov, Elements of Applied Bifurcation Theorey, Springer-Verlag, New York, NY, USA, 3rd edition, 2004.

[10] X. Liu and D. Xiao, "Complex dynamic behaviors of a discrete-time predator-prey system," Chaos, Solitons \& Fractals, vol. 32, no. 1, pp. 80-94, 2007.

[11] A. Q. Khan, J. Ma, and D. Xiao, "Bifurcations of a two-dimensional discrete time plant-herbivore system," Communications in Nonlinear Science and Numerical Simulation, vol. 39, pp. 185-198, 2016.

[12] Z. Hu, Z. Teng, and L. Zhang, "Stability and bifurcation analysis of a discrete predator-prey model with nonmonotonic functional response," Nonlinear Analysis: Real World Applications, vol. 12, no. 4, pp. 2356-2377, 2011.

[13] Z. Jing and J. Yang, "Bifurcation and chaos in discrete-time predator-prey system," Chaos, Solitons \& Fractals, vol. 27, no. 1, pp. 259-277, 2006.

[14] C.-H. Zhang, X.-P. Yan, and G.-H. Cui, "Hopf bifurcations in a predator-prey system with a discrete delay and a distributed delay," Nonlinear Analysis: Real World Applications, vol. 11, no. 5, pp. 4141-4153, 2010.

[15] M. Sen, M. Banerjee, and A. Morozov, "Bifurcation analysis of a ratio-dependent prey-predator model with the Allee effect," Ecological Complexity, vol. 11, pp. 12-27, 2012.

[16] A. Q. Khan and M. N. Qureshi, "Global dynamics and bifurcations analysis of a two-dimensional discrete-time LotkaVolterra model," Complexity, vol. 2018, Article ID 7101505, 18 pages, 2018. 